DOI: $10.47460 /$ uct.v24i107.409

\title{
ESTRATEGIAS DE GESTIÓN EMPRESARIAL: UN ACERCAMIENTO A LA PLANEACIÓN SISTEMÁTICA
}

\author{
Monserrat Salas ${ }^{1}$. Christian Jijón ${ }^{2}$ y Kléver Moreno ${ }^{3}$ \\ monserratsalas@hotmail.es ${ }^{1}$; davicho030796@gmail.com ${ }^{2}$; kleveramoreno@uta.edu.ec ${ }^{3}$ \\ https://orcid.org/0000-0002-0750-8179 ' , https://orcid.org/0000-0002-5802-5716 2, \\ https://orcid.org/0000-0001-9870-8821 ${ }^{3}$ \\ Universidad Técnica de Ambato \\ Ambato - Ecuador
}

Recibido (02/10/20), Aceptado (20/11/20)

\begin{abstract}
Resumen: Las estrategias de gestión empresarial tienen como finalidad crear y mantener ventajas competitivas a través de un plan de acción de manera que sea sostenible en el tiempo, sin embargo el éxito de las mismas dependerá de la mejora continua en el modelo de gestión empresarial y así aumentar la productividad, competitividad, y eficiencia. El objetivo del estudio es determinar la heterogeneidad del modelo de gestión estratégica empresarial mediante el meta-análisis de dimensiones. El diseño metodológico tuvo un enfoque cuantitativo de corte histórico-hermenéutico, pues el levantamiento de información se realizó mediante una modalidad bibliográfica. El método aplicado fue el meta-análisis que permitió calcular el tamaño de la variación teórica. El resultado principal fue que la heterogeneidad se presentó con mayorénfasis en la estrategia de costos con relación a las otras dimensiones. Se concluyó que el incorrecto manejo de las estrategias empresarial depende del déficit existente en la planeación sistemática.
\end{abstract}

Palabras Clave: Gestión empresarial, meta-análisis, planeación sistemática

\section{BUSINESS MANAGEMENT STRATEGIES: AN APPROACH TO SYSTEMATIC PLANNING}

\begin{abstract}
The business management strategies are intended to create and maintain competitive advantages through an action plan so that it is sustainable over time, however their success will depend on continuous improvement in the business management model and thus increase productivity, competitiveness, and efficiency. The objective of the study is to determine the heterogeneity of the business strategic management model through the meta-analysis of dimensions. The methodological design had a quantitative approach of historical-hermeneutical nature, since the information gathering was carried out through a bibliographic modality. The method applied was the meta-analysis that allowed calculating the size of the theoretical variation. The main result was that heterogeneity was presented with a greater emphasis on the cost strategy in relation to the other dimensions. It was concluded that the incorrect management of business strategies depends on the existing deficit in systematic planning.
\end{abstract}

Keywords: Business management, meta-analysis, systematic planning 


\section{I.INTRODUCCIÓN}

El concepto de gestión empresarial, ha evolucionado en la medida que el hombre ha avanzado en la consecución de nuevas tecnologías y relaciones para el mejoramiento de nuevos productos y servicios [1]. Por lo tanto, las estrategias se definen como el ajuste que una empresa realiza entre sus habilidades y recursos internos con las oportunidades y riesgos creados por sus factores externos, las mismas que, pueden variar entre cinco matrices de cualquier secuencia: FODA, PEEA, BCG, IE y la estrategia principal. Mientras que, la planeación sistemática comprende la complejidad en el mundo al desenvolverse pues, esta se basa de subsistemas y al fallar una no puede funcionar lo demás en conjunto [2].

El objeto de estudio propio, la metodología de investigación y la teoría desarrollada es la particularidad epistémica de los estudios organizacionales, pues, su peculiaridad se basa en la multidisciplinariedad y transdisciplinariedad, por lo que, al tratar de conocer, percibir e intervenir en la empresa, fundamenta la naturaleza de un nuevo campo científico, por lo que, la planificación es el proceso que determina con anticipación las acciones que se deben desarrollar y ejecutar antes de actuar, para alcanzar los objetivos deseados [3].

Por lo tanto, la gestión empresarial ya no es un argumento de carácter local ni tampoco nacional, las distancias de nuestro mundo, en la evolución de nuevas sociedades de información y conocimiento, deben considerarse como un asunto de índole mundial, para el desarrollo de nuevas estrategias en la permanencia en el mercado y la satisfacción a sus clientes en cualquier parte del mundo [4].

En Latinoamérica se garantiza la productividad, rendimiento y competitividad de las compañías, pues, la gestión empresarial recae en los directores, gerentes, consultores y especialistas [5]. Según la revista FORBES [6] evidencia que, la acción diaria se enfoca en el logro de los objetivos de la compañía, por lo tanto, se los cumple con eficacia, eficiencia y efectividad. Mientras que, el crecimiento de las empresas se evidencia es capaz de generar recursos que mantienen su actividad y generan un excedente, es decir que, el crecimiento de una empresa depende del $85 \%$ de una buena gestión empresarial [5].

En el Ecuador, el entorno se desarrolla de manera dinámica, compleja y diversa, puesto que, requiere de un monitoreo permanente debido a la gran cantidad de cambios y problemas que enfrenta [7]. Constantemente, este evoluciona, se adapta, transforma y se convierte en competencia, no obstante, algunos casos fracasan [8]. Esta visión solo se comparte con el apoyo del sector empresarial, pues, deben ser capaces de competir a cualquier nivel y cambios de procesos, los mismos que, se verán regidos a implementar modelos, metodologías o estrategias de planeación sistemática.

La provincia de Tungurahua según el censo nacional económico del 2010 es la tercera provincia que posee micro y pequeñas empresas de orden familiar $(8,1 \%)$ [9]. Las mismas que, presentan problema de sucesión de mando, ya que al ser conformadas de manera empírica no logran pasar a la tercera generación debido a una inadecuada administración y aplicación de estrategias organizacionales, lo que ha conllevado a no cumplir sus objetivos empresariales [10].

El planteamiento del modelo de gestión empresarial expone diversas dimensiones de las cuales se establecieron la estrategia de costos, estrategia operativa, estrategia de inversión y estrategia comercial. La estrategia operativa tiene mayor predominancia dentro del análisis literario. Mientras que, la estrategia de costos tiene divergencia en el estudio de estrategias empresariales.

Por lo tanto, la estrategia es la búsqueda deliberada de un plan de acción que una organización realiza para crear y mantener ventajas competitivas, las mismas que, son características diferenciales de una compañía respecto a otras que confieren la capacidad para alcanzar rendimientos superiores a ellas, de manera sostenible en el tiempo [11]. Para lo cual, se realizó un análisis sistemático de revisión bibliográfica de estudios publicados que contribuyeron al desarrollo de este escrito.

El estudio se direccionó bajo cuatro (4) hipótesis orientadas hacia la homogeneidad:

a) la estrategia de costos incide en el modelo de gestión estratégica empresarial.

b) la estrategia de operativa incide en el modelo de gestión estratégica empresarial.

c) estrategia de inversión incide en el modelo de gestión estratégica empresarial.

d) estrategia comercial incide en el modelo de gestión estratégica empresarial.

\section{II.DESARROLLO}

\section{A.Estrategia empresarial}

La forma en la que la organización interactúa con su entorno desarrolla los principales recursos y medios para alcanzar sus objetivos [12]. Por lo tanto, se lo asemeja como un desempeño de gestión consciente y explícito de forma anticipada, pues su finalidad es asegurar el logro de los objetivos determinados por la empresa [13]. Por ende, se puede decir que, la estrategia es 
la búsqueda deliberada de un plan de acción que realiza para crear y mantener ventajas competitivas [14]. Se entiende que la ventaja competitiva es una de las características diferenciales que le confiere la capacidad para alcanzar rendimientos superiores de manera sostenible en el tiempo.

Por lo general, para hacer que la empresa sea efectiva y competitiva en el mercado debe satisfacer las necesidades de grupos o segmentos de clientes, sobresalir ante la competencia, ofrecer productos o servicios destinados a dichos segmentos, defenderse de movimientos competitivos de los rivales, tomar acciones en función de las tendencias de la industria, cambios en la economía o movimientos políticos y sociales, entre otros [15]. Todos ellos implementados en una estrategia que procure la sobrevivencia de la organización tanto de manera interna como externa.

\section{B.Antecedentes en la gestión empresarial}

La evolución histórica de la gestión empresarial, tiene un desenvolvimiento de ideas a nivel cultural en oriente y occidente, alcanzando el desarrollo del hombre en cada uno de los sistemas sociales [15]. La toma decisiones ha evolucionado al analizar sus cuatro funciones claves para el desarrollo de mando a nivel empresarial, como lo son; planificar, organizar, dirigir y controlar, por consiguiente, una gestión y persona dinámica en el mundo empresarial en el desenvolvimiento de un entorno y mercado competitivo y productivo a una escala mundial [16].

La gran diversidad de teorías, enfoques y pensadores de las alternativas estratégicas, aunque, cada empresa debe definir su estrategia en función del mercado y de sus propias características, existen diversas alternativas genéricas que se pueden adoptar al período que se requiere.

\section{C.Gestión empresarial}

La gestión empresarial es un proceso estratégico, dependiente y de control en el manejo de los recursos de la empresa y así aumentar la productividad, competitividad, y eficiencia [17]. Por otro lado, la gestión empresarial, también conocida como ingeniería de gestión empresarial, se diferencia de la administración de empresas al combinar tanto la gestión estratégica como la aplicación de la tecnología e innovación [18].

Según el economista y autor Joseph Schumpeter (1883-1950), citado por [19] asegura que, la gestión empresarial garantiza que la oferta cubra la demanda mediante la destrucción creativa, o sea, con la innovación constante para aumentar la productividad y la competitividad.
Sin embargo, si decidimos establecer una definición más concreta en cuanto a lo referido para el Comercio Europeo en productos y servicios podemos considerar en este caso que la gestión empresarial debe conceptualizarse en relación a la calidad, seguridad, responsabilidad, confianza, competencias, protección al trabajador, etc. Todos ellos factores imprescindibles que harán crecer a una empresa generadora de productos y servicios [20].

La gestión empresarial es la brújula que guía el camino de un negocio, su estrategia y finalidad. Al final, es un recurso imprescindible en cualquier sistema capitalista basado en inversiones y retorno [21]. Por lo tanto, muchos profesionales que emprenden un negocio se encuentran perdidos ante este panorama, y creen que el rendimiento de una empresa solo depende de factores ajenos a ellos como la suerte o el azar [22]. Sin embargo, los especialistas en economía y gestión saben de sobra que un negocio sin estrategia no es nada, porque tarde o temprano las anomalías del mercado terminan por impactar en su rendimiento

No hay que olvidar que, una empresa con éxito debe mantenerse focalizada en que sus gestiones sean las más acertadas. pues, en el caso del sector privado, tanto la inversión de los socios como el balance de ganancias con la facturación, son dos frentes a los que debe prestársele especial atención.

Esta es una de las principales diferencias entre la empresa pública y privada, porque en la primera de ellas, los índices de rentabilidad y productividad no son tan importantes como en el sector particular, donde llevar a cabo una correcta gestión empresarial se convierte en algo obligatorio [23].

Las estrategias genéricas de Michael Porter tiene como objetivo lograr una ventaja competitiva de largo plazo, penetración del mercado, obtener mayor rentabilidad en el mercado. Para ello se nombran a tres estrategias competitivas como lo son: liderazgo en costos, diferenciación y enfoque [24].

\section{D.Liderazgo de costos}

La reducción de costos a partir del control riguroso de los gastos fijos (costo de producción, distribución, administrativo y financiamiento) o gastos variables (materia prima, mano de obra directa, depreciación) que pueden afectar el valor unitario del producto final. El objetivo de la estrategia es obtener mayor participación en el mercado ofreciendo un producto de igual o mayor calidad a bajo costo frente a la competencia [25]. Sin embargo, la estrategia es factible en mercados amplios, es decir, en producción a gran escala, pues un producto estandarizado en calidad y precio tiene mayor acepta- 
ción por múltiples oferente y organizaciones [26]. Por lo tanto, en mercados reducidos las ganancias no serían mayoritarias debido al margen de utilidad presente en cada producto.

\section{E.Diferenciación}

La percepción de algo único ya sea en el diseño, imagen, marca o tecnología del producto o servicio, así como su post venta. La estrategia tiene factibilidad cuando brinda protección en contra de la competencia, es decir, originalidad y calidad en sus productos, pues se mencionan que los clientes son leales a la marca minimizando la susceptibilidad del precio [27]. La diferenciación puede impedir una gran participación en el mercado, sin embargo, logra la fidelidad de los clientes y genera una posición estable frente a la competencia [28]. Por lo tanto, dicha estrategia no es relativa con precios bajos o semejantes a la competencia pues lo que busca exclusividad.

\section{F.Enfoque}

Se centra en un segmento de mercado en específico, pues su objetivo es producir bienes o servicios que logren satisfacer necesidades de un grupo limitado [29] . En comparación con las anteriores estrategias que buscan desarrollar productos o servicios de calidad en mercados extensos, el enfoque antepone la rentabilidad del volumen de ventas, la atención personalizada de clientes, adecuación de productos en nichos pequeños, analiza el desarrollo y penetración del mercado, donde el control exhaustivo es eficiente por pertenecer a un segmento y no a un mercado general [30].

El presente modelo se estructura mediante las estrategias genéricas de Porter y la calidad como estrategia competida presentando en cuatro (4) dimensiones, tomando en cuenta diversos factores que pueden llegar a influir en la estrategia de gestión empresarial. Cabe recalcar que dicho modelo se está basado en puntos clave que propone el investigador. La misma que, consta de: 1) Estrategia de costos, 2) Estrategia operativa, 3) Estrategia de inversión y 4) Estrategia comercial (ver tabla I).

La primera dimensión se enfoca en la actividad industrial que se presenta como los costos de producción, distribución, administración y financiamiento. La segunda dimensión se centra en el talento humano, tecnología e innovación, servicios y productos de las empresas a su manejo operativo.

La tercera dimensión corresponde a la competitividad de las empresas toando en cuanta las subdimensiones del análisis situacional, desarrollo de productos, desarrollo de mercados y la penetración del mercado.
La cuarta dimensión toma en cuenta los elementos de la entrega del producto, el trato con el cliente y su servicio post venta (ver tabla I).

\section{Tabla I. Estrategia de gestión empresarial}

\begin{tabular}{|c|c|c|}
\hline \multirow{11}{*}{ 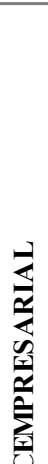 } & DIMENSION & SUBDIMENSION \\
\hline & \multirow{8}{*}{ Estrategia de costos } & Costos de \\
\hline & & Producción \\
\hline & & Costos de \\
\hline & & Distribución \\
\hline & & Costos de \\
\hline & & Administración \\
\hline & & Costos de \\
\hline & & Financiamiento \\
\hline & \multirow{5}{*}{ Estrategia operativa } & Talento humano \\
\hline & & Tecnología e \\
\hline \multirow{4}{*}{ 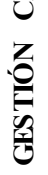 } & & innovación \\
\hline & & Servicios \\
\hline & & Productos \\
\hline & \multirow{4}{*}{$\begin{array}{l}\text { Estrategia de } \\
\text { inversión }\end{array}$} & Análisis situacional \\
\hline \multirow{6}{*}{ 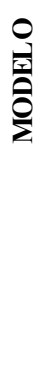 } & & $\begin{array}{l}\text { Desarrollo de } \\
\text { productos }\end{array}$ \\
\hline & & $\begin{array}{l}\text { Desarrollo de } \\
\text { mercados }\end{array}$ \\
\hline & & $\begin{array}{l}\text { Penetración de } \\
\text { mercado }\end{array}$ \\
\hline & \multirow{3}{*}{ Estrategia comercial } & Estandarización \\
\hline & & Servicio al cliente \\
\hline & & Postventa \\
\hline
\end{tabular}

\section{III.METODOLOGÍA}

Para demostrar la amplitud de la investigación se partió de la selección de investigaciones de tipo empírico y de revisión sistemática donde se caracterizó y estructuró la relevancia del estudio de la Estrategia de gestión empresarial. Se planteó un modelo teórico de gestión estratégica a partir de la dirección de Michel Porter que permitirá garantizar la funcionalidad de cada área del entorno empresarial [31] .

La aplicación de criterios de inclusión y exclusión permitió seleccionar estudios mediante un análisis crítico a través de diferentes filtros. Los mismos que, se aplicaron en una muestra no probabilística de 75 artículos categorizados como revisión sistemática y empírica. El estudio supone una investigación exploratoria, pues es basada en un enfoque cuantitativo de corte histórico-hermenéutico, ya que, se realizó el levantamiento de información mediante una modalidad bibliográfica con el fin de rectificar los lineamientos teóricos que tienen un sesgo de error, pues no han sido modificadas en una delimitada línea de tiempo. De forma que, mantienen un cierto grado de deficiencias.

El método aplicado fue el meta-análisis que permitió 
calcular el tamaño del efecto de variación teórica para obtener mayor precisión del problema del modelo de estudio, mediante la observación de artículos publicados que muestran resultados negativos o insignificantes. Para la minería y análisis de los escritos, se estableció la estrategia de búsqueda que incorporó dos fases: la primera constó de criterios de inclusión, donde se tomó en cuenta la base indexada de los artículos tomados de Scopus, Scielo, Latindex y libros; palabras clave acerca del tema de investigación, identificador del objeto digital (DOI); así como, el idioma de los artículos que fueron en español, inglés y portugués; la selección de los artículos fue de orden empírico y sistemático. Por otra parte, los criterios de exclusión tuvieron tres elementos: revistas predadoras, definidas como revistas con fines lucrativos sin el rigor científico necesario, blog o páginas web y estudios publicados antes del año 2015.

\section{IV.RESULTADOS}

Los resultados fueron presentados de manera cronológica según la estrategia de búsqueda presentada en los criterios de inclusión 1) Base indexada: los resultados de búsqueda obtenidos en Scielo, Scopus, Latindex y Libros. 2) Idioma: el origen y el país de los documentos en el que fueron publicados. 3) Orientación: el tipo de investigación de los documentos que verificas si son de estudio sistemático o empírico. 4) Frecuencia: el año de publicación que tuvo mayor relevancia presentada en diagrama de dispersión. 5) diagrama de bosque: que permitió medir la hipótesis de la investigación. Además, se presentó la evaluación de cada dimensión según el modelo de gestión empresarial con el propósito de poner en evidencia el factor que ha sido descuidado por los investigadores al momento de implementar las estrategias de negocio.

De acuerdo al criterio de indización de artículos, se determinó que, el mayor porcentaje de artículos indexados pertenecen a la base científica Scopus con un 37\% y Scielo con $28 \%$. No obstante, el artículo denominado Ranking de revistas científicas en Latinoamérica mediante el índice h: estudio de caso Colombia demostró que, los estándares de calidad editorial del índice Scopus son altamente confiables, pues su reconocimiento internacional contribuye con los atributos de calidad lingüística, originalidad y creatividad. Por tal motivo, los temas con relación a las estrategias empresariales son mayormente publicados en Scopus.

Dentro del análisis de revisión literaria se determinó que el porcentaje de prevalencia de idioma es el español, pues predominó con un 78\%. Por lo tanto, el mayor número de revistas son de origen latinoamericano predominado por Colombia con el $20 \%$, seguido de
Ecuador con el 16\% y Brasil con el 12\%. Por otro lado, la estrategia de gestión empresarial en Europa específicamente en España apenas un 13\%. Pues, en su estudio denominado Estrategias empresariales españolas en un mundo interdependientes se aseguró que, las relaciones hispano-latinoamericanas son cualitativamente superiores debido a la proximidad del mercado, la similitud de los modelos de consumo y nivel de integración europea existente.

La información verificada indicó que el 91\% de los artículos recopilados y analizados corresponden a estudios de resultados empíricos y apenas el 9\% son de revisión sistemática. Lo que significó, que no existe lineamientos de mejora con respecto a la literatura, pues los estudios tienden a centrarse en resultados prácticos y no en una adecuación de ejes teóricos relacionados con las variables de estudio (ver figura 1).

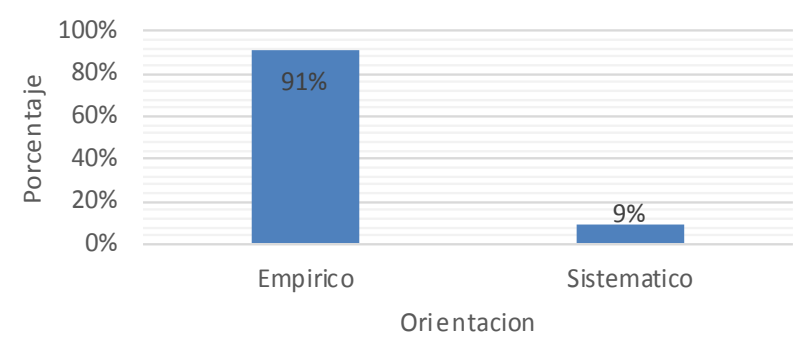

\section{Figura 1. Orientación}

Fuente. Elaboración propia

Dentro de la minería y análisis de información de escritos publicados se observó que entre los años 19952008 los investigadores no han profundizado el estudio de estrategias empresariales, pues apenas en el año 1992 Norton y Kaplan integran las métricas de identificación para las estrategias empresariales, por lo que se puede decir que los estudios hasta el año 2008 son basados en las métricas presentadas por los autores [32] . El tema de estudio con relación a los artículos publicados entre los años 2010-2020 presentaron un incremento, dado a que los autores Koontz, Weihrich y Cannice en el año 2012 ejecutaron los procesos y acciones que cumplen con la estrategia de gestión a través de un ciclo continuo. Por lo tanto, el aumento de estudios presentados hasta el año 2020 se deben al enfoque que dan los autores con respecto a la ejecución de la misma (ver figura 2) [33]. 


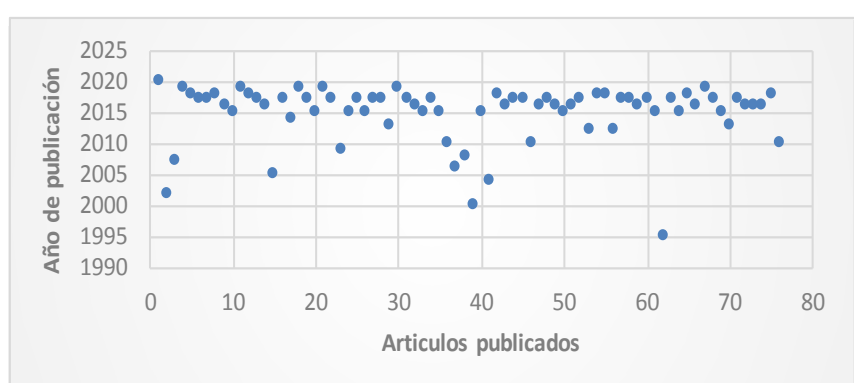

Figura 2. Frecuencia de publicación por año

Fuente. Elaboración propia

El meta-análisis tiene el propósito de evidenciar el área que ha tenido sublevación o traducido como heterogeneidad dentro del modelo de gestión estratégica empresarial donde se analizó las dimensiones: a) estrategia de costos, b) estrategia operativa, c) estrategia de inversión y d) estrategia comercial (Ver figura 3). Dentro del análisis global, la estrategia de costos presentó heterogeneidad con relación a las otras dimensiones, ya que se aplicó la prueba de heterogeneidad de Dersimonian y Laird's con un valor $\mathrm{p}=0,4063$. Lo que significó, la exclusión del cuadrante de homogeneidad, es decir, que tenga relación con el estudio. De igual forma, en el estudio de Bosch, se demostró que los altos índices de inversión son vitales durante el crecimiento de una empresa, pues, es indispensable para dar a conocer el producto y generar mayor énfasis en el desarrollo de nuevas tecnologías, para que el producto se mantenga en el mercado. Por otro lado, el estudio denominado Análisis y gestión estratégica de costos menciona que, el objetivo de la estrategia de costos es mantener la mejora continua de bienes o servicios. Sin embargo, el uso de la mismas sin una combinación integral con otras estrategias como: el liderazgo en costos, la diferenciación de los productos y el análisis de las ventajas competitivas, podría llevar el declive de la empresa.

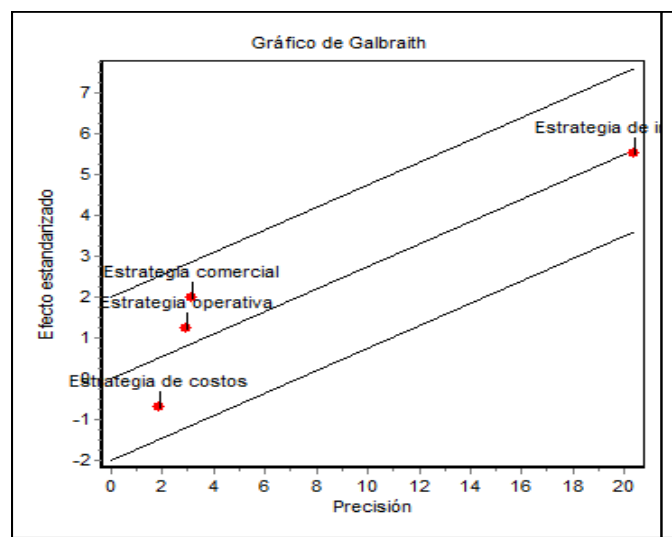

Figura 3. Meta-análisis

Fuente. Elaboración propia

En el análisis de la figura 4, la estrategia de costos según la prueba de heterogeneidad de Dersimonian y Laird's determinó un valor $\mathrm{p}=0,3591$. Es decir, las estrategias de costos se mantienen en el cuadrante de homogeneidad con el modelo analizado. Por lo tanto, Boscan, M. y Samdrea, M., en su estudio denominado Estrategias de financiamiento para el desarrollo endóge-

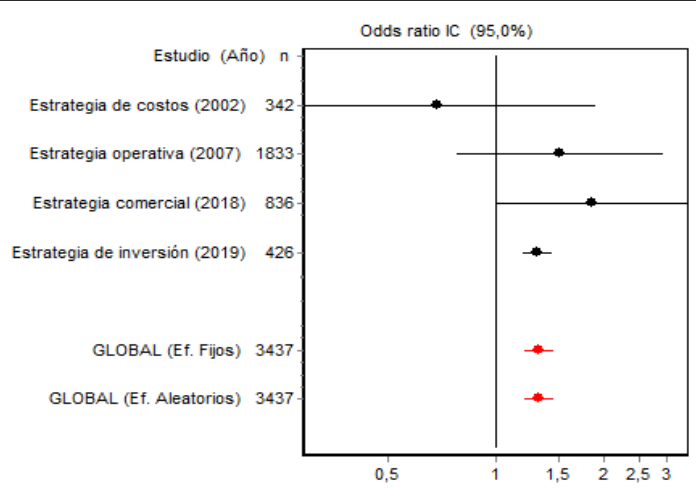

no demostraron que la disposición de las estrategias de financiamiento, son funcionales a corto plazo [34]. De manera que, recurrir a pagos por adelantado y cancelación de facturas, satisface los requerimientos de capital de trabajo, es decir, que se amplía el financiamiento, pues al acudir a una banca privada con montos mayoritarios puede afectar al sector administrativo. 


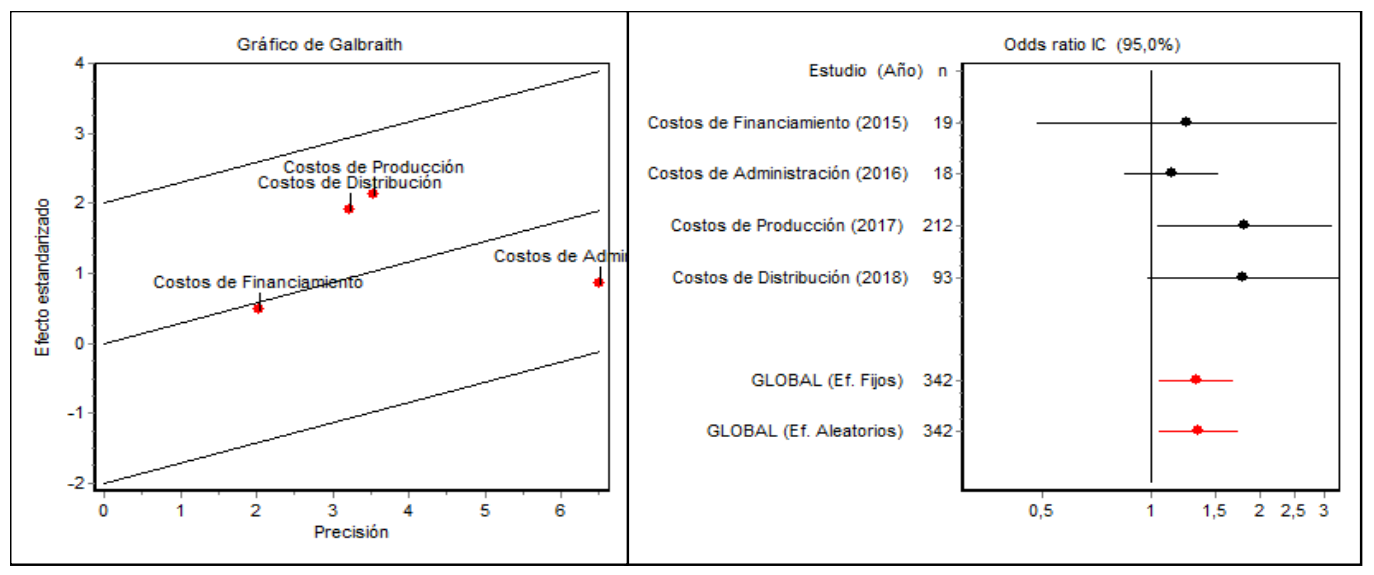

\section{Figura 4. Meta-análisis. Estrategia Costos}

Fuente. Elaboración propia

La estrategia operativa según la prueba de heterogeneidad de Dersimonian y Laird's se obtuvo un valor $\mathrm{p}=0.738$. Lo que quiso decir, que las estrategias operativas mantienen homogeneidad con el modelo de estudio, por tal razón, se alineo al cuadrante de homogeneidad con excepción de los servicios (Ver figura 5). De modo que, el estudio denominado Fundamentos de la gestión tecnológica e innovación asegura que las estrategias de negocio proponen la adquisición de la tecnología y recursos humanos necesarios para el desarrollo de los productos de calidad que permiten anticipar la evolución y mantener una ventaja competitiva, aunque signifique el descuido de otros aspectos corporativos como procesos de post-venta o servicios aledaños [35].

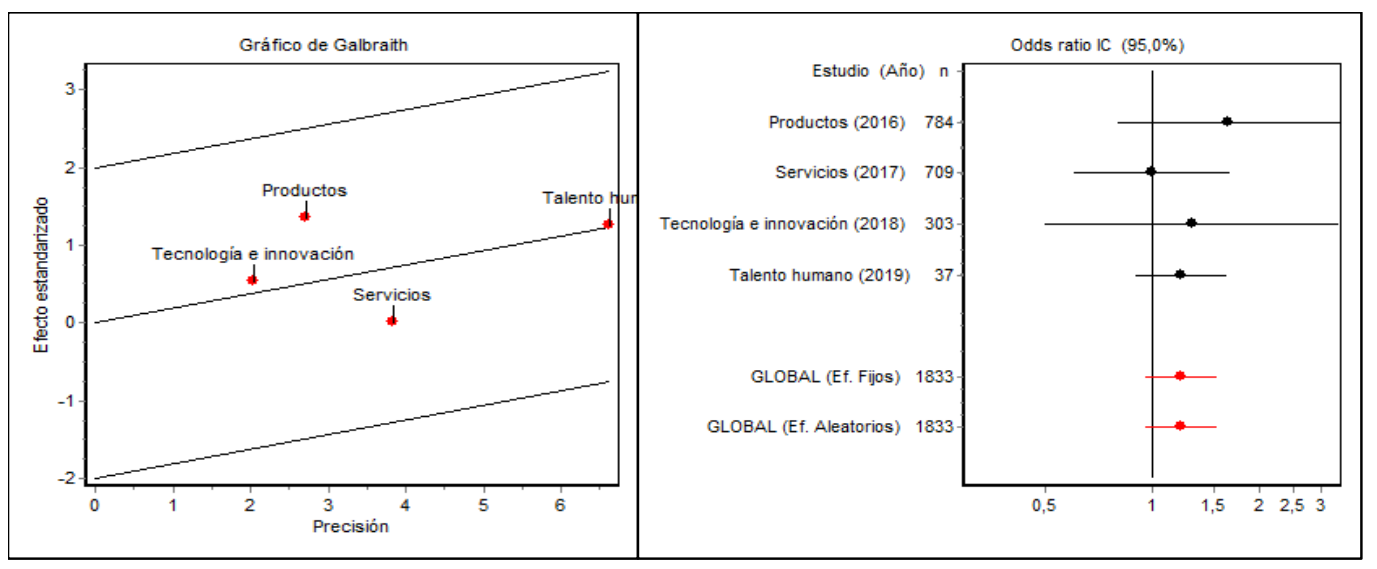

Figura 5. Meta-análisis. Estrategia de Operativa

Fuente. Elaboración propia

El análisis de la figura 6, demostró que, en la estrategia de inversión según la prueba de heterogeneidad de Dersimonian y Laird's se obtuvo un valor $p=0,05$. De forma que, si existe heterogeneidad en las estrategias de inversión con el modelo de gestión estratégica. Sin embargo, uno de los factores destacados como heterogéneo dentro del modelo de estudio es el desarrollo de mercado (Ver figura 10). De igual modo, Maldonado

G. y Erazo M., en su estudio denominado El reto de la planificación estratégica en las Pymes aseguraron que, la inexistencia de planeación estratégica dentro de estas organizaciones hace que se deteriore su gestión empresarial y proyectos de inversión sobre los cuales mantienen una trayectoria a largo plazo. Dicho de otra forma, pierden estabilidad y perspectiva de crecimiento al no ajustarse a las necesidades del mercado. 


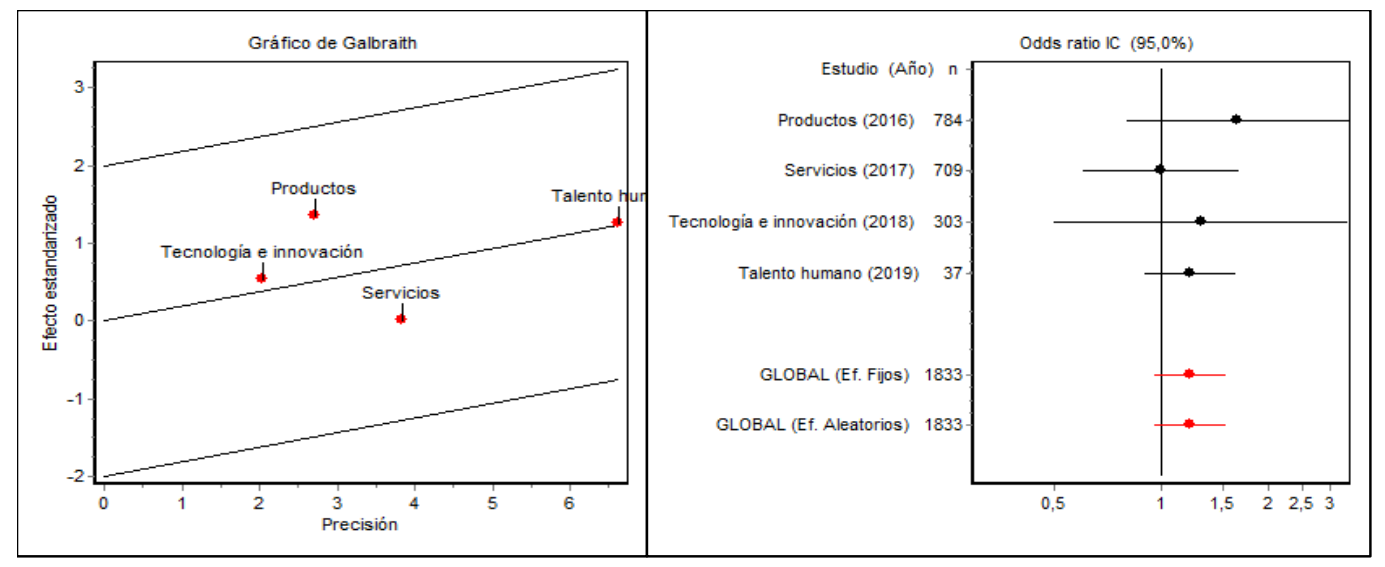

Figura 6. Meta-análisis. Estrategia de Inversión

Fuente. Elaboración propia

La estrategia de comercial según la prueba de heterogeneidad de Dersimonian y Laird's determinó que existe heterogeneidad con un valor $\mathrm{p} \leq 0,04$. No obstante, se visualizó que existen factores excluidos del cuadrante de homogeneidad entre aquellas fue la estandarización (Ver figura 7). De igual modo, el estudio Anna León F. denominado la gestión post venta como instrumento de fidelización en una empresa comercializadora de maquinaria estableció que, la fidelización de los clientes potenciales se constituyó por servicio al cliente y post-venta de calidad, dicho esto, el estudio denominado Estandarizar o no estandarizar: esa es la pregunta, asevera que, la variación del beneficio contable estandarizado dependerá del tamaño de la empresa, pues las variaciones en empresas grandes son irrelevantes, sin embargo, en una empresa pequeña se distorsiona el beneficio contable [36].
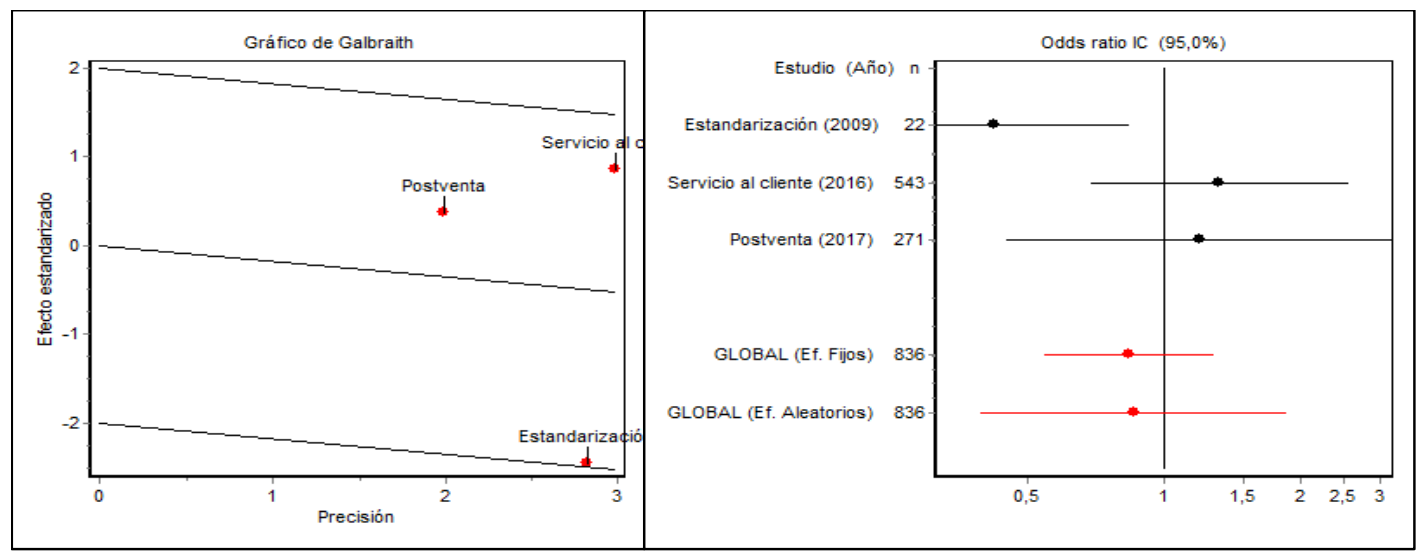

Figura 7. Meta-análisis. Estrategia Comercial

Fuente. Elaboración propia

\section{V.CONCLUSIONES}

Se concluyó que, mediante la prueba de heterogeneidad de Dersimonian y Laird's en el meta-análisis, la estrategia de costos presento heterogeneidad con relación a las otras dimensiones, sin embargo, la exclusión de los cuadrantes en costos de financiamiento y administrativo, es decir, que sus áreas no son atendidas de manera idónea y no incurre en la gestión estratégica empresarial. Por otra parte, en la estrategia operativa, el meta-análisis mostró una gran heterogeneidad en los servicios, por tal razón, la profundización del área se debe a un problema metodológico de las empresas al no tener en cuenta un enfoque global de las estrategias.

Mientras que, la heterogeneidad presentada en las estrategias de inversión por la prueba de Dersimonian y Laird's demostró que, la falta de planeación estratégica deteriora los proyectos de inversión y pierdan el desarrollo un mercado potencial, siendo este uno de los factores en que se deberían poner énfasis en la gestión empresarial. Además, la estrategia comercial en la inci- 
dencia en la gestión empresarial de acuerdo con el meta-análisis estableció que, la heterogeneidad presentada en la estandarización se dependerá del tamaño de la empresa en el que la aplica pues, el beneficio contable no es igual a una PYMES con una empresa Multinacional.

\section{REFERENCIAS}

[1]A. Villacis, S. Prado, J. Cedeño y L. Morales, «Adinistración una herramienta de la planificación,» Revista Ceintifica Mundo de la Investigación y El Conocimiento, pp. 737-751, 2018.

[2]H. Contreras, «Estrategia Empresarial,» In ColeÇāo Gestāo Empresarial ,pp. 15-26, 2002.

[3]M. Suarez, «Gestión empresarial: un paradigma del siglo XXI,» Revista Científica FIPCAEC (Fomento de La Investigación y Publicación En Ciencias Administrativas, Económicas y Contables), pp. 44-64, 2018.

[4]B. Peña, P. del Henar y G. Gonzalez, «Estartegias inteligentes de comunicación interna y gestión empresarial,» Revista Opción, pp. 1-23, 2015.

[5]S. Lee, C. Hahn, M. Rhee, J. Song, Y. Chen y G. Lu, «Plan de negocios y planeacion estrategica empresarial en el siglo XXI.,» Journal of Chemical Information and Modeling, pp. 1689-1699, 2012.

[6]L. T. Hidalgo, A. Castro y B. Loor, «Formación en emprendimiento en el Ecuador. Pertinencia y fundamentación epistemológica,» Revista Espacios, pp. 1-12, 2018.

[7]S. Jaramillo, «Planeación estratégica y su aorte al desarrollo empresarial,» Espiritu emprendedor TES, pp. 64-73, 2019.

[8]Y. González, «La planeación emprearial: Un acercamiento conceptual,» Contribuciones a La Economía, p. $11,2010$.

[9]INEC, «Directorio de empresas y establecimientos,» Quito, 2018.

[10]F. Pozos y M. Acosta, "Analysis and relevance in business development.,» Revista Científica Pensamiento y Gestión, pp. 184-202, 2016.

[11]M. González, «Gestión estratégica como herramienta para promover la competitividad de las empresas del sector logístico del departamento del Atlántico, Colombia,» Espacios, pp. 38-51, 2017.

[12]J. Garcia, S. Duran, E. Cardeño, R. Prieto, E. Garcia y A. Paz, «Proceso de planificación estratégica: Etapas ejecutadas en pequeñas y medianas empresas para optimizar la competitividad,» Espacios, pp. 38-52, 2017.

[13]C. Jannuzi, C. Sugahara y J. Sousa, «Estrategia empresarial e innovación tecnológica de las industrias brasileñas,» Revista de Ivestigación Academica, pp. 65-84, 2015.

[14]L. Joia y M. de Olivera, «PersonalizaÇāo ou co-
dicaÇāo? Avaliando estratégias de foco em gestāo do conhecimento,» Scielo, pp. 3-15, 2007.

[15]S. Bravo, J. Azcárate, A. Rojas y C. Narváez, "Comparative Analysis of Strategies and Competitiveness Between Mipymes Associates and No Associates.,» Espacios, pp. 1-24, 2017.

[16]R. Molina, O. González y A. Mendivelso, «Revisión epistemológica del gobierno corporativo y la Responsabilidad Social Empresarial.,» Contexto, pp. 4356, 2017.

[17]F. Cusi, «Planificación estratégica con enfoque en los agronegocios,» Revista de la Carrera de Ingenieria Agronómica, pp. 885-893, 2017.

[18]S. Pinto, P. Ramírez y E. Grandón, «Antecedentes del Éxito de los sistemas de planificación de recursos empresariales en las grandes empresas chilenas: Un modelo factorial exploratorio,» Información Tecnologica, pp. 139-146, 2017.

[19]C. Ramió, «Teoría de la Organización y Administración Pública.,» de Teoria de la Organización y Administración Pública, Colombia, 2010, p. 304.

[20]F. Hernández, O. Flores y G. Isaac, «Propuesta de un modelo integral de competitividad económica para las empresas del municipio de Nuevo Laredo (Proposal of an integral model of economic competitiveness for the companies of Nuevo Laredo).,» Innovaciones de Negocios, pp. 67-81, 2005.

[21]M. Beltran, V. Parrales y G. Ledesma, «El Buyer Persona como factor clave entre las tendencias en Gestión Empresarial,» Recimundo, pp. 659-681, 2019.

[22]N. Khabazi, N. Feghi y S. Iranzadeh, «A comprehensive model for energy management strategies in coordination with manufacturing and organization strategies and its effect on energy management performance.,» Cogent Business and Management, pp. 18-29, 2018.

[23]C. Lopez, « Identificación de escenarios prospectivos y líneas de acción para el desarrollo del sector de motocicletas colombiano,» Espacios, pp. 14-27, 2017.

[24]B. Husted y D. Allen, «Strategic Corporate Social Responsibility and Value Creation Among Large Firms.,» Proceedings og the International Association for Business and Society, pp. 215-223, 2004.

[25]S. Bravo, J. Azcárate y A. Rojas, «Comparative Analysis of Strategies and Competitiveness Between Mipymes Associates and No Associates,» Desarrollo Gerencial, pp. 102-121, 2015.

[26]M. Larrañaga y Ortega, «La planeación estratégica de las micro y pequeñas empresas de alojamiento temporal en Cuernavaca, Morelos , México.,» Vincula Tegica, pp. 323-331, 2018.

[27]A. Mora y E. Acevedo, «Revisión sistemática de 
literatura sobre la internacionalización de la empresa PYME en Colombia,» Espacios, p. 2017, 13-39.

[28]V. Novoa, «La competitividad en las Instituciones de Educación Superior: aplicación de filosofías de gestión empresarial. Gestión del proceso de negocio,» Economía y Desarrollo, pp. 166-181, 2016.

[29]D. Escado y A. Hurtado, «Influencia de los estilos de liderazgo en el desempeño de las empresas exportadoras colombianas,» Estudios Gerenciales, pp. 137145, 2016.

[30]V. Duro y V. Gilart, «La competitividad en las Insituciones de Educacion Superior: aplicacón de filosofía de gestión empresarial. Gestión de proceso de negocio,» Economia y Desarrollo, pp. 1-17, 2016.

[31]E. Castro, «Las estrategias competitivas y su importancia en la buena gestión de las empresas,» Ciencias Económicas , pp. 247-276, 2010.

[32]M. Malgioglio, C. Carazay, D. Suardi, G. Bertolino, T. Díaz y D. Fernández, «Distintos enfoques del capital intelectual,» de Distintos enfoques del capital intelectual, Lima, 2002, pp. 1-43.

[33]L. Lopez, M. Parra y G. Rubio, «Habilidades gerenciales y su relación con la perdurabilidad de las empresas: estudios de caso empíricos en Florencia e Ibagué (Colombia),» Espacios, vol. 40, pp. 5-20, 2018.

[34]M. Boscán y M. Sandrea, «Estrategias de financiamiento para el desarrollo endógeno,» Telos, vol. 11, $\mathrm{n}^{\mathrm{o}}$ 3, pp. 402-417, 2009.

[35]J. Gallejo, «Fundamentos de la gestión tecnologica e innovación,» Tecno Logicas, $\mathrm{n}^{\mathrm{o}}$ 15, pp. 113-131, 2015.

[36]D. Mannucci, «la gestión post venta como instrumento de fidelización en una empresa comercializadora de maquinaria,» 2016. [En línea]. Available: http://200.37.102.150/bitstream/USIL/3590/1/2016_ León-Genit.pdf. [Último acceso: 2020]. 